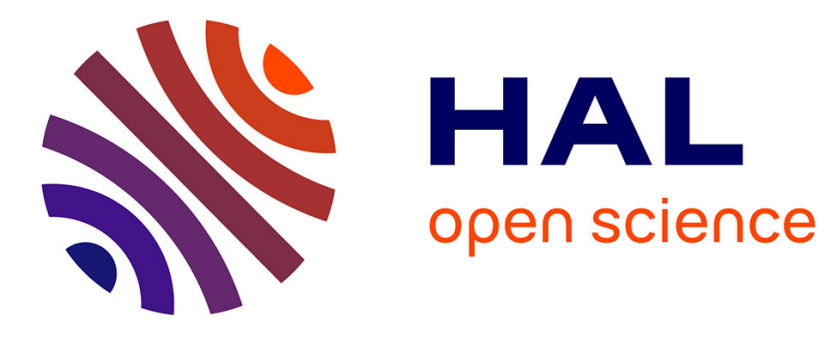

\title{
Les lombalgies chroniques : symptôme ou pas symptôme ?
}

Patrick Martin-Mattera, . Ly-Thanh-Huê, François Garnier, Jean-François Huez, Vincent Benoist

\section{To cite this version:}

Patrick Martin-Mattera, . Ly-Thanh-Huê, François Garnier, Jean-François Huez, Vincent Benoist. Les lombalgies chroniques : symptôme ou pas symptôme ?. L'Évolution Psychiatrique, 2010, 75 (4), pp.607-620. 10.1016/j.evopsy.2010.09.005 . hal-03389915

\section{HAL Id: hal-03389915 https://univ-angers.hal.science/hal-03389915}

Submitted on 21 Oct 2021

HAL is a multi-disciplinary open access archive for the deposit and dissemination of scientific research documents, whether they are published or not. The documents may come from teaching and research institutions in France or abroad, or from public or private research centers.
L'archive ouverte pluridisciplinaire HAL, est destinée au dépôt et à la diffusion de documents scientifiques de niveau recherche, publiés ou non, émanant des établissements d'enseignement et de recherche français ou étrangers, des laboratoires publics ou privés. 


\title{
Psychiatrie aux limites \\ Les lombalgies chroniques : symptôme ou pas symptôme?
}

\section{Chronic low back pain: Symptom or no symptom?}

\author{
Patrick Martin-Mattera $^{\mathrm{a}, *, \mathrm{~b}}$, Ly-Thanh-Huê ${ }^{\mathrm{c}}$, François Garnier $^{\mathrm{d}}$, \\ Jean-François Huez ${ }^{d}$, Vincent Benoist ${ }^{\mathrm{e}}$ \\ a Psychologue, psychanalyste, professeur de psychopathologie, centre de recherche de l'Institut de psychologie et \\ sociologie appliquées (CERIPSA), université catholique de l'Ouest (UCO), \\ 3, place André-Leroy, 49008 Angers cedex 01, France \\ ${ }^{\mathrm{b}}$ EA 2646, laboratoire de psychologie Processus de pensée et interventions (PPI), université d'Angers, \\ 49000 Angers, France \\ ${ }^{\mathrm{c}}$ Docteur en psychopathologie fondamentale et psychanalyse, psychiatre, CERIPSA, UCO, 3, place André-Leroy, \\ 49008 Angers cedex 01, France \\ d Professeur (assistant) de médecine générale, département de médecine générale, faculté de médecine, \\ rue Haute-de-Reculée, 49045 Angers cedex 01, France \\ e Docteur en psychopathologie, CERIPSA, UCO, 3, place André-Leroy, 49008 Angers cedex 01, France \\ Reçu le 14 octobre 2009 \\ Disponible sur Internet le 13 octobre 2010
}

\section{Résumé}

Cet article étudie un aspect psychologique impliqué dans certaines affections somatiques, ici les lombalgies chroniques. Plusieurs cas de patients lombalgiques reçus en entretiens psychothérapiques montrent que le statut de la douleur évolue selon que le sujet lui accorde ou non une valeur de symptôme, au sens psychanalytique. Le «choix» de ce statut est personnel, subjectif, inconscient, et impacte l'évolution de la lombalgie. Après avoir défini le symptôme au sens médical et au sens psychanalytique, et avoir rappelé la distinction entre l'hystérie et la psychosomatique, les auteurs présentent leur conception des rapports corps et psyché selon le modèle de la bande de Möbius utilisée par Lacan pour définir les rapports entre le conscient et l'inconscient. Il est cependant illusoire, en médecine générale, de pouvoir amener tous les patients à faire de leur lombalgie un symptôme "psychanalytique». Le contexte et le cadre ne sont pas les mêmes car le patient qui va consulter son médecin n'en attend pas la même chose que celui qui va consulter un psychologue, un

\footnotetext{
论 Toute référence à cet article doit porter mention : Martin-Mattera P, Ly-Thanh-huê, Garnier F, Huez JF, Benoist V. Les lombalgies chroniques: symptôme ou pas symptôme ? Evol psychiatr 2005; 75 (4).

* Auteur correspondant. 66, rue du Docteur-Guichard, 49000 Angers, France.

Adresse e-mail : martinmattera@wanadoo.fr (P. Martin-Mattera).
} 
psychanalyste ou un psychiatre. Néanmoins, l'ouverture des uns et des autres à la possibilité d'une évolution du patient fondée sur un changement de sa position subjective peut être un avantage important, car ce qui est en jeu dans le processus de la chronicisation n'est pas seulement la disparition pure et simple de la douleur, mais la modification préalable de sa fonction pour le sujet, c'est-à-dire le passage de la fonction première de la douleur comme signal de danger à celle qui favorise l'élaboration d'un sens subjectif. Et pour cela, faut-il encore que ce sujet, comme les praticiens concernés, lui reconnaissent cette seconde fonction.

() 2010 Elsevier Masson SAS. Tous droits réservés.

Mots clés : Lombalgie ; Maladie chronique ; Médecine générale ; Symptôme; Psychanalyse ; Médecine psychosomatique

\begin{abstract}
This article examines a psychological aspect involved in some somatic disorders, in particular chronic back pain. Several cases of patients with low back pain received in psychotherapeutic interviews show that the status of pain changes, depending on whether they consider that it has symptom value or not, in the psychoanalytical sense. The "choice" of this status is personal, subjective, unconscious, and has an impact on the development of LBP. After defining the symptom in the medical sense and in the psychoanalytical sense, and pointing out the distinction between hysteria and psychosomatic, the authors present their approach to the link between the body and the psyche according to the Möbius strip model used by Lacan to define the relationship between the conscious and the unconscious. However, it is illusory, that general medicine can lead all patients to make their back pain a "psychoanalytical" symptom. The context and the setting are different because the patient who consults his doctor does not expect the same as he who goes to a psychologist, a psychoanalyst or a psychiatrist. Nevertheless, that people open to the possibility of an evolution of the patient based on a change in his subjective position may be an important advantage, because what is in play in the process of chronicity is not only the disappearance of chronic pain, but the change of its function for the subject, that is to say the way from the first function of pain as dangers' signal to the second that permits an elaboration of a subjective sense. And for that, it is necessary that the patient as well as the practitioners concerned recognize this second function.
\end{abstract}

(c) 2010 Elsevier Masson SAS. All rights reserved.

Keywords: Chronic low back pain; Chronic disease; General medicine; Symptom; Psychoanalysis; Psychosomatic

Nous proposons ici d'étudier un des aspects psychologiques pouvant être impliqués dans certaines affections somatiques. Dans le cas présent, la population prise en considération est celle de patients souffrant de lombalgie chronique. Nous verrons, à travers plusieurs exemples concrets, que le statut de la douleur évolue selon que le patient lui accorde ou non une valeur de symptôme, au sens psychanalytique du terme.

\title{
1. Position du problème en médecine générale
}

La lombalgie commune est un symptôme fréquent en médecine générale, mais seulement $8 \%$ des patients présentant ce symptôme passeront à la chronicité ${ }^{1}$. La lombalgie chronique commune (non spécifique) est définie par une douleur lombaire évoluant depuis plus de trois mois ${ }^{2}$. Le médecin généraliste est fréquemment confronté à ce passage à la chronicité sans possibilité d'en apprécier tous les facteurs déterminants. Les facteurs psychologiques, sociaux et environnemen-

${ }^{1}$ Poireaudeau S., Lefèvre-Colau M.-M., Fayad F., Rannou F., Revel M. Lombalgies. In [1] p. 295-319.

2 [1] et Agence Nationale d'Accréditation et d'Évaluation en Santé [2]. 
taux semblent plus importants dans la pérennisation et la récidive de la lombalgie que les facteurs physiques et mécaniques.

De nombreuses études ont permis de proposer au médecin praticien une prise en charge permettant :

- de dépister le risque de chronicité en mesurant la perception du patient quant à son incapacité et sa probabilité de retour à ses activités habituelles ${ }^{3}$;

- puis d'identifier les obstacles en cas de probabilité faible de reprise d'activité, qu'ils soient:

- des obstacles cliniques,

- des obstacles liés au travail,

○ des obstacles psychologiques ou sociaux.

Les deux premiers ont fait l'objet de beaucoup de recherches et les résolutions proposées semblent satisfaisantes. Les obstacles psychologiques et sociaux sont plus difficiles à maîtriser même si certains ont pu être identifiés et validés : la détresse psychologique, la dépression, les peurs et croyances, le catastrophisme, la somatisation.

Il est alors possible de faire le constat suivant:

- le médecin, dans sa pratique, explore peu les facteurs psychologiques et sociaux ${ }^{4}$ (en dehors de ceux liés au travail);

- les réponses biomédicales proposées aux patients lombalgiques sont insuffisantes aussi bien pour les médecins que pour les patients les mettant tous deux en situation d'impasse ;

- les propositions d'ordre biomédical faites habituellement ne permettent pas de résoudre toutes les situations.

Dans ce contexte, le présent écrit se veut être une contribution à la saisie d'un aspect important du registre psychologique dans l'évolution de certaines lombalgies.

\section{Symptôme}

Le mot symptôme n'a pas le même sens en médecine et en psychanalyse. En médecine, le symptôme résulte de ce qu'exprime un malade, par ses dires ou par son corps, ouvrant le champ des maladies possibles indiquées par ce symptôme. Un mal de ventre est un symptôme qui peut évoquer un trouble fonctionnel ou de multiples maladies somatiques. En psychanalyse, et de fait dans une large part de la psychopathologie influencée par elle, le symptôme est plutôt ce que Freud définissait comme une «formation de compromis » entre les exigences du ça, du surmoi et du monde extérieur, formation portée par le moi. Une compulsion obsessionnelle du type vérification de la fermeture d'une serrure pourrait réaliser ainsi par exemple un acte sexuel symbolisé par l'introduction et le retrait successif de la clé dans la serrure et sa punition surmoïque par la contrainte imposée de la répétition excessive de ces gestes. Cet exemple

\footnotetext{
${ }^{3}$ Par exemple grâce aux échelles de mesure suivantes : modèle pronostique des maux de dos, échelle d'incapacité du dos du Québec, etc.; cette évaluation est proposée à la quatrième semaine ou immédiatement si le patient a un passé de lombalgie avec incapacité.

${ }^{4}$ Pour un apport à ce propos, voir : Benoist V., Martin-Mattera P., Ly-Thanh-Huê, Huez J.F., Garnier F. La chronicité chez les patients lombalgiques, In: [3].
} 
est caricatural, il indique que le symptôme en psychanalyse possède $d u$ sens, mais il faut ajouter qu'il ne s'agit jamais d'un sens en soi, d'un sens absolu et unique. Le sens du symptôme, c'est le sujet qui le lui donne et même ainsi, ce sens est voué à évoluer, à se transformer sans cesse au cours du travail analytique à la faveur de l'interprétation. Freud indique ceci : «les symptômes ont pour intention soit une satisfaction sexuelle, soit une défense contre cette dernière; [...] ils sont [...] des résultats de compromis issus de l'interférence de deux tendances opposées et représentent aussi bien le refoulé que le refoulant, qui a coopéré à leur apparition ${ }^{5}$. Lacan, en 1966, épure quant à lui la pensée freudienne et affirme que «ce que Freud a toujours dit du symptôme, c'est qu'en lui-même le symptôme est jouissance ${ }^{6}$. Ainsi, le symptôme, dans le champ analytique, est à la fois une plainte et une satisfaction dont le sujet ne connaît ni la source ni l'objet et qui en définitive se révèle à lui comme souffrance. Car la jouissance n'est en effet pas le plaisir, elle en excède les limites et s'impose au sujet malgré lui. La jouissance en réfère toujours à l'interdit, dont le modèle ici est le tabou fondamental de l'inceste maternel. Dans le texte précédemment évoqué, Freud précisait que si la névrose obsessionnelle accentuait dans le symptôme son côté «défensif» contre la sexualité, dans l'hystérie en revanche, c'est le côté satisfaction sexuelle qui était privilégié. Qu'est-ce à dire concernant l'hystérie?

\section{Hystérie ou psychosomatique}

Nous avons remarqué que le terme d'hystérie est souvent source de confusion entre praticiens de registres différents, comme la médecine et la psychopathologie. Dans la même veine se situe celui de psychosomatique, généralement source de contresens encore plus importants. Il s'agit alors, pour avancer dans notre propos, de situer rapidement les limites au sein desquelles nous utilisons ici ces deux termes.

L'hystérie ne correspond pas à la simulation ni au faire semblant d'être malade, bien qu'une certaine démonstrativité du patient puisse être souvent observée. Elle correspond plutôt à ce que l'on a l'habitude de considérer comme des manifestations de la conversion somatique, autrement dit d'une expression involontaire du corps venue à la place d'une parole qui ne peut s'énoncer aussi bien que d'une satisfaction interdite. C'est pourquoi l'on peut réaffirmer que le statut du symptôme est bien celui d'une jouissance. Le symptôme hystérique «parle » en quelque sorte et peut être interprété.

La notion de psychosomatique est beaucoup plus délicate. «Elle traduit les rapports complexes qu'entretient le sujet moderne avec les maux qui l'atteignent dans son corps et importunent son esprit», écrit Pascal-Henri Keller ([5], p. 10-1). Disons d'abord que la psychosomatique n'est pas l'hystérie. Ensuite, et dans notre perspective, elle ne correspond pas à un ensemble particulier de maladies, dont l'étiologie serait principalement psychique, ni à un type particulier de malades, comme par exemple les alexithymiques ${ }^{7}$, ou les personnes relevant d'une pensée opératoire, selon l'expression de Marty et De M'Uzan [7]. Pierre Marty considérait, dans son livre Les mouvements individuels de vie et de mort, que les névroses et les psychoses, parce qu'elles sont selon lui «organisées dans leurs défenses mentales », échappent aux maladies psychosomatiques, tandis que les névroses de caractère et de comportement, peu organisées dans leurs

\footnotetext{
${ }^{5}$ Freud S. Leçons d'introduction à la psychanalyse. In: CEuvres complètes, T. XIV ([4], p. 311).

${ }^{6}$ Lacan J. Séminaire XIII, L'objet de la psychanalyse, séance du 27 avril 1966. Inédit.

7 «Alexithymia», selon John Nemiah et Peter Sifneos, [6].
} 
défenses, seraient un terrain particulièrement propice d'éclosion des maladies pychosomatiques ([8], p. 97-8). Bien que nous partagions avec Marty l'idée d'abandonner «le principe du dualisme psyché-soma» ([8], p. 16), nous ne nous situons pas dans cette perspective qui isole des «structures » plus ou moins sensibles à la maladie psychosomatique. La notion de psychosomatique relève pour nous de la nécessaire prise en compte du sujet dans toute affection, fût-elle somatique, qui le touche. La psychanalyse nous enseigne que nul n'est étranger à ce qui lui arrive dans son corps ou dans son esprit et que, justement, les limites que nous posons entre le corps et l'esprit, ne sont pas si évidentes du fait que le corps auquel nous avons affaire n'est pas «simple»: est-il corps symbolique, comme celui de l'hystérique, qui parle à qui peut ou non l'entendre, est-il corps de la réalité effective, tel que la médecine en comprend et en infléchit parfois le fonctionnement, est-il enfin corps réel qui échappe au sujet, s'autonomise, dérape et l'entraîne finalement dans la mort? Sami-Ali se demandait en 1977: "comment définir le corps dans son appartenance ambiguë au réel et à l'imaginaire?» ([9], p. 82). Cette question est ici au cœur de notre clinique puisqu'il s'est agi pour nous de déterminer justement le statut de la douleur et de la plainte lombalgiques. Comme nous le verrons plus loin, nous posons que ce statut est fonction de la position subjective et aboutit soit à un enkystement psychosomatique où cette douleur ne fait pas symptôme, soit à la constitution d'un symptôme hystérique dans lequel le sujet «parle» à travers son corps. Cela dit, on peut penser que la psychosomatique correspond, dans son aspect le plus moderne, à la prise en considération de cette dimension du sujet dans l'être malade, quelle que soit la maladie: elle se situe comme un questionnement sur la « responsabilité » du sujet dans ce qui lui arrive. Que l'on considère spécifiquement l'existence de «phénomènes corporels », ou «phénomènes psychosomatiques » selon l'expression utilisée par Jean Guir qui a développé un courant d'étude psychosomatique d'obédience lacanienne [10], ces phénomènes corporels correspondent à ce que Freud appelait les névroses actuelles, et sont un élément important d'éclairage de la notion de psychosomatique, parce que cela permet d'accentuer le fait que ces phénomènes ne parlent pas, ne s'interprètent pas, et surtout ne font pas symptômes, contrairement à l'hystérie : «Les symptômes des névroses actuelles [. . .] n'ont aucun sens, aucune signification psychique », écrit Freud ([4], p. 400). Mais cela ne doit pas occulter le fait que n'importe quelle maladie peut s'imposer au sujet précisément de cette manière, et ceci consiste alors à se dédouaner de toute responsabilité en la matière et en définitive revient à s'y dénier comme sujet. Jean Guir affirme très justement dans Psychosomatique et cancer, «qu'il n'y aurait donc plus, en pareil cas, de dialectique du sujet» ([10], p. 4). Notre hypothèse défend l'idée que même lorsque cette dialectique du sujet a disparu, le sujet lui-même n'a pas pour autant été complètement évacué, n'a pas purement et simplement disparu. Il y a en effet dans toute affection somatique, qu'on la considère comme hystérique ou psychosomatique, un positionnement - différent - du sujet, à partir duquel il est possible de penser la place de cette affection dans l'économie psychique et somatique, ce qui permet parfois de transformer l'équilibre de cette économie.

Dans la perspective que nous développons ici, la position du sujet peut donc être soit «psychosomatique», ce qui veut dire que le sujet considère que sa douleur résulte de causes principalement extérieures qui échappent totalement à sa maîtrise, soit «névrotique » (ou hystérique) et il présentera alors un symptôme qui s'inscrira pour lui dans son histoire en prenant sens. Nous soulignons alors le fait que, dans notre vue des choses, le sujet malade n'est pas forcément prisonnier de son positionnement subjectif, psychosomatique ou hystérique, mais qu'il peut être amené à évoluer, lors d'un travail psychothérapique, vers une «hystérisation» de sa douleur en faisant de sa plainte une parole. Précisons que le terme d'hystérisation ne signifie pas ici structure hystérique, mais qu'il faut y entendre plutôt 
l'idée d'un mouvement de subjectivation, d'une mobilisation de la parole à l'adresse d'un autre.

C'est ce que nous allons maintenant présenter à travers des éléments de la clinique de patients lombalgiques.

\section{La lombalgie chronique : symptôme ou pas symptôme?}

Quel est le statut de la lombalgie chez chaque sujet lombalgique ? Pour lui, pour elle, la lombalgie fait-elle ou non symptôme, et à quelles conditions? À partir de plusieurs entretiens psychanalytiques effectués auprès de patients lombalgiques, nous avons constaté que la lombalgie, très souvent, n'apparaît pas seule, mais prend place, dans le discours du sujet, au sein d'une constellation d'autres plaintes ou souffrances, de nature somatique, psychique, ou relationnelle.

On trouvera ci-dessous quatre vignettes cliniques qui en montrent la multiplicité. Ces vignettes sont «graduées ». Elles tendent à montrer chez les patients différents stades de leur pensée sur la maladie et de leur positionnement subjectif. Elles présentent des situations, comme celle d'Aristide, qui vont du registre psychosomatique où aucun symptôme n'est élaboré, jusqu'au registre de l'hystérisation dans lequel la douleur lombalgique prend place dans l'économie psychique par un faisceau de sens, comme dans l'observation d'Isophane, en passant par une situation « intermédiaire », celle de Tiphaine. Une quatrième vignette plus développée, celle de Loryanne, montrera, grâce à l'usage du modèle de la bande de Möbius, comment penser ce passage éventuel de la psychosomatique à l'hystérie.

\section{Aristide}

Aristide a 53 ans, ses lombalgies débutent dans l'enfance. À l'âge de dix ans, il porte des semelles. À 17 ans, première sciatique. "C'est devenu chronique je l'ai su dès le départ, je ne voyais pas de fin. On ne sait pas quoi y faire. Mais quand on est jeune on ne se rend pas compte. On ne sait pas ce que c'est que le temps». À 32 ans, des crises de cruralgie et de cervicalgie apparaissent. «Je compense au niveau du dos». À 51 ans, une crise de sciatique qui se prolonge au-delà d'une semaine l'amène en milieu hospitalier durant neuf jours. Il y reçoit un traitement à la morphine et deux infiltrations de cortisone. Il évoque des crises de tachycardie de l'âge de 15 ans jusqu'à 23 ans. "Même sans effort ça montait à 220 puis ça redescendait. Je n'ai plus fait de sport. Ca s'est arrêté à l'âge de 23 ans».

De son état actuel, il dit: «je ne souffre plus psychologiquement. Ca me bloque, il faut l'accepter. Ca ne me stresse plus. Je n'ai pas un caractère à me stresser, j'ai appris à vivre avec. Je suis d'un naturel calme. Fataliste peut-être: c'est comme ça. Il faut accepter mais en espérant ne pas avoir trop mal. Je fais du jardin doucement, je marche beaucoup». Il note un épisode douloureux dans sa vie. À l'âge de 23 ans, il perd son père lors d'un accident de la route. Sa mère avait 55 ans et elle a très mal supporté cette perte.

Pour Aristide, la lombalgie reste extérieure dans sa survenue, elle est le résultat d'une fatalité ou d'un mauvais hasard de vie, où s'enchaînent les conséquences malheureuses de ce handicap. Il ne parvient pas à élaborer sa lombalgie en symptôme, en tout cas pas encore, et s'il énonce les difficultés de sa vie, la mort prématurée de son père, les difficultés de sa mère, il ne tisse pas les liens de sens qui pourraient lui faire occuper la place centrale dans les aléas de son existence. Sa problématique apparaît pour le moment comme une série de phénomènes somatiques non subjectivés, qu'il n'investit pas de sens et qu'il accepte par force, même s'il 
ne renonce pas à combattre en maintenant des activités physiques destinées à surmonter son handicap.

\section{Tiphaine}

Tiphaine, 56 ans, a «des douleurs tout le temps, depuis des années ». «C'est pire depuis trois ou quatre ans. Depuis le cou, les épaules...». Elle a subi une opération «plastie» dans une épaule, elle a de l'arthrose dans les pouces, au talon, aux genoux, au dos. . Elle est en mi-temps thérapeutique et bénéficie de séances de kinésithérapie depuis qu'elle a subi cette opération de l'épaule. Elle a eu ses premières douleurs à 24 ans, «quand j'attendais mon fils » (Tiphaine a un fils de 31 ans et une fille de 29 ans). «Maintenant j'ai des vertèbres abîmées », et lors de la naissance de sa fille, «ça a repris». Tiphaine souffre également de dépression depuis 1991 (elle avait 38/39 ans): "quand j'ai eu 35, 38 ans, je me suis dit ma mère est morte à cet âge-là... J'ai été arrêtée un an et demi, j'étais dans le fond du gouffre. Après, ça a été, jusqu'au moment où on a su la maladie de mon mari. Il est mort dix ans après, mais pas à cause de la maladie, parce qu'il a fait une chute. Et puis il y a eu la maladie de mon fils» (maladie neuromusculaire évolutive comme le père). Par ailleurs, Tiphaine sait être de nature anxieuse, crispée, et pense que cela accentue ses douleurs: "j'ai toujours vécu, pas pour moi, mais dans la maladie. Ma mère a souffert du cœur, elle est décédée très jeune [à l'âge de $35 \mathrm{ans}$ ], j'en ai souffert. Mon mari avait cette maladie neurologique [mari décédé il y a quatre ans] et mon fils l'a aussi. Tout ça fait que je ne suis jamais vraiment bien». "Quand je regarde la télévision, je me dis "décontracte-toi», mais je n'y arrive pas. J'ai mal aussi dans mon lit. J'ai des problèmes d'endormissement ». De son histoire, Tiphaine rappelle que son père «avait plein de douleurs », et avait un traitement pour cela, «par piqûres». Tiphaine se souvient de lui, montant les escaliers à quatre pattes quand la douleur était trop forte «surtout vers la fin». Sur le plan familial, Tiphaine dit: «j'ai un frère qui ne va pas mieux que moi, il a souvent des douleurs dans le dos; mes enfants sont pareils, ma fille, mon fils, ils ont mal dans le dos, ils ont des ceintures. (...) La sœur de mon père c'est pareil, elle est pleine de douleurs ».

Tiphaine parvient à relier entre elles les difficultés de sa vie, mais pour autant ne réussit pas à se situer pleinement comme actrice de ce qui lui arrive. Elle subit ces événements et développe deux importantes voies de souffrance, la lombalgie et la dépression, qu'elle intègre dans une «filiation » de la douleur, celle de son père «plein de douleurs », la mort de ses proches, sa mère, morte alors qu'elle-même avait seulement 12 ans, son mari mort de maladie neuromusculaire, son fils qui en est aussi atteint. Son interprétation de la lombalgie est à la fois psychique et « mécanique », puisqu'elle pense que son anxiété et ses crispations accentuent ses douleurs. L'élaboration des difficultés rencontrées montre que même s'il y a une inscription psychique complexe des représentations associées à ces difficultés, cela en reste à une plainte portant sur des événements sur lesquels Tiphaine pense n'avoir aucune prise. Même si la problématique de Tiphaine est «mentalisée » sur bien des points importants de son existence, celle-ci évite d'y inclure ce qui relève de la lombalgie elle-même. Elle n'élabore donc pas encore de symptôme à cet endroit.

\section{Isophane}

Isophane est une femme de 45 ans, notaire. Les premiers lumbagos «chroniques », dit-elle, sont survenus il y a dix ans, avec d'abord des épisodes d'une semaine à dix jours, puis une hernie discale et l'opération consécutive, qui a entraîné un arrêt de travail de plusieurs mois. Cela s'est produit au moment où elle a déménagé avec son nouveau mari. C'était pendant des vacances 
chez sa sœur, en marchant: des douleurs intolérables se déclenchent. Isophane a vécu dix ans avec son premier mari, puis elle divorce et la situation est conflictuelle. Mais «quand j'avais des problèmes avec mon ancien mari, je n'avais pas de maux de reins », dit-elle. Les premières souffrances ont débuté avec la naissance de sa deuxième fille (douleurs de dos). Elle dit que son enfance a été «radieuse » avec des parents très attentifs. Mais après, cela s'est gâté : "mon père était très présent, trop sans doute, puis il a fait une dépression, il y a eu une cassure, c'était un patriarche, il a fait une psychose maniaco-dépressive ». Il a voulu interdire le mariage de la sœur avec drame, violences, menaces de suicide. Puis Isophane s'est installée dans une autre région pour son métier et a culpabilisé de laisser sa mère avec son père, qu'elle va peu voir car il n'a jamais accepté qu'elle parte (toujours menaces de suicide). Elle a eu des problèmes avec lui quand elle s'est mariée. Les lombalgies se sont déclenchées à la mort d'amis très chers : une amie décédée d'un cancer, et ensuite ça a été l'enfant de cette amie, puis un collègue qui s'est suicidé. "La première série de lombalgies, ça a été la séparation d'avec mon premier mari, une forme de deuil». "Chaque fois que j'ai perdu des amis, il y a eu un supplément d'exigence de qualité de vie à donner aux autres, et j'ai pas mal d'amis qui sont partis très jeunes». «Je suis très exigeante, mais la hernie discale m'a contrainte à lâcher prise ».

Lorsque Isophane dit: "La première série de lombalgies, ça a été la séparation d'avec mon premier mari, une forme de deuil », elle ne dit certainement pas la vérité historique des événements «séparation » et «première lombalgie », mais elle constitue le sens de son histoire dans l'aprèscoup d'une association d'idées qui s'impose à elle. Elle fait donc de sa lombalgie un symptôme «qui parle », à la condition qu'elle l'adresse à quelqu'un. La lombalgie lui permet ainsi de dire autre chose, de faire surgir en elle les signifiants qui fondent son existence et qui la vectorisent: le père maniaco-dépressif, la mort brutale d'amis, l'importance de la sœur, des ruptures, etc. La problématique d'Isophane s'inscrit ainsi dans une dimension psychique d'élaboration, où la lombalgie occupe une place subjective importante qui ouvre la voie à des possibilités de changement diversifiées.

\section{Loryanne}

Une dernière observation, celle de Loryanne, nous fournit l'occasion de développer un exemple de passage entre une problématique psychosomatique et une dynamique hystérique et d'en constater également les aléas et les difficultés. Le cas de cette patiente montre que même si, d'une part, la structure générale de la personnalité est névrotique, avec production de symptômes, faisant sens pour elle, à tel ou tel endroit de son organisation psychique, cela, d'autre part, ne la protège nullement de difficultés somatiques caractérisées par une absence de production symptomatique : à la place elle subit des phénomènes «corporels » qui lui restent extérieurs.

Loryanne est née en 1954, son mari décède en septembre 2007 d'un cancer du pancréas à l'âge de 54 ans, et elle consulte pour un état dépressif en mars 2008. "Il y a ce mensonge entre nous, on ne lui a jamais dit qu'il était perdu». «Je ne lui ai pas dit au revoir. Il ne parlait plus. Je me suis endormie et à cinq heures du matin il était mort». Dans ses antécédents, on trouve des lombalgies chroniques depuis l'âge de 21 ans, une sciatique après la naissance des enfants, (elle a une fille de 30 ans, un fils de 29 ans et une fille de 28 ans), et une reprise des lombalgies depuis le décès du mari. Elle présente aussi une tendinite calcifiante aux deux épaules, des brûlures œsophagiennes, un reflux gastro-œsophagien depuis l'âge de 40 ans, avec accentuation de la symptomatologie depuis le décès du mari : brûlures œsophagiennes diurnes, glossodynies. Elle a subi une cholécystectomie en 2006 et a une hypercholestérolémie depuis 2006. De plus 
elle souffre d'une fissure anale et de lithiase rénale. Loryanne fait des crises d'angoisse : «j'ai l'impression qu'il va revenir [il s'agit de son mari]. Il n'a jamais dit qu'il allait mourir, il n'a jamais prononcé le mot "mort" ». Elle pense de fait beaucoup à son mari, à la vie passée avec lui, aux activités de chorale et de peinture qu'il appréciait. Elle range ses affaires, ses tableaux, et les donne aux amis et aux enfants. Loryanne se plaint de contractures musculaires. "J'en ai toujours eu. Maintenant avec la dépression c'est pire. J'ai mal partout dans tout le dos». Et elle ajoute: "Tout ce qui m'arrive réveille en moi une vieille douleur». Quand elle en vient à se plaindre des douleurs œsophagiennes, elle en montre le trajet: «Ca me brûle de partout...», et dit: «Ça ne m'étonne pas que tout ça m'arrive. J'attrape tout en ce moment». Elle parle de l'absence concrète de la chaleur du corps du mari et dort avec son petit-fils de quatre ans quand il vient à la maison. Le traitement est le suivant : antidépresseur et anxiolytique par le médecin traitant, séances de kinésithérapie, arrêt de travail. Consultations en gastroentérologie (bilan : pH métrie, manométrie œsophagienne «non contributives »), en rhumatologie, au centre antidouleur (prescription d'abord d'antiépileptique [clonazepam], puis d'antidépresseur [duloxétine] à visée antalgique), et parallèlement elle est adressée par son médecin traitant en psychothérapie d'inspiration psychanalytique.

Loryanne souffre de façon multiple, elle est la "proie» de phénomènes corporels qu'elle ne tente pas tous de comprendre et auxquels elle ne prête pas sens. Pourtant, parallèlement, elle produit des associations d'idées qui ouvrent la voie d'une élaboration potentielle de symptômes qui prendront sens pour elle. Cependant, cette élaboration psychique se heurte encore à un apparent clivage entre les différents plans de sa souffrance, somatique ou bien psychique, clivage qui sera mis en question par le travail psychothérapique qu'elle a entrepris.

\section{Application möbienne}

Cette dernière observation permet de remarquer un enchaînement de « causalité circulaire », qui indique chez cette patiente une alternance entre souffrance psychique et souffrance somatique. La Fig. 1, ci-dessous, implique une circularité entre les causes psychiques et les causes somatiques des symptômes: il peut y avoir, pour un même sujet à deux moments différents et pour une même plainte ou un même énoncé, deux causes différentes. D'une part, deux atteintes somatiques lésionnelles de statut subjectif différent, psychosomatique ou hystérique, peuvent produire les mêmes douleurs psychiques, les mêmes affects et les mêmes représentations. D'autre part, les mêmes douleurs psychiques, les mêmes affects et les mêmes représentations peuvent survenir à partir d'atteintes lésionnelles réelles (position psychosomatique du sujet), ou se produire sans lésions constatées (position hystérique du sujet).

Il y a ainsi, dans cet exemple, une continuité entre les phénomènes somatiques et psychiques, au sein de laquelle resterait encore à interroger plus précisément les points de passage d'un registre à l'autre. Il semble qu'une tension psychique liée au deuil vienne augmenter les tensions dans le corps, les contractures locales existantes, déstabilise ainsi l'équilibre acquis et réveille des lombalgies anciennes. Mais force est de constater aussi une discontinuité entre les phénomènes observés, révélant une autonomie des phénomènes somatiques et psychiques qui valent aussi pour leur propre compte: les phénomènes lésionnels, issus d'une position psychosomatique du sujet, ou les symptômes psychiques de conversion en relation avec la structure d'origine, ici la structure hystérique. Dans le contexte de cette structure, se formulent ainsi les mêmes représentations, affects, douleurs psychiques, qu'il y ait des atteintes lésionnelles réelles ou bien qu'il n'y ait pas de lésions constatées. 


\section{Atteintes corporelles}

(lésions colonne vertébrale, oesophagite)

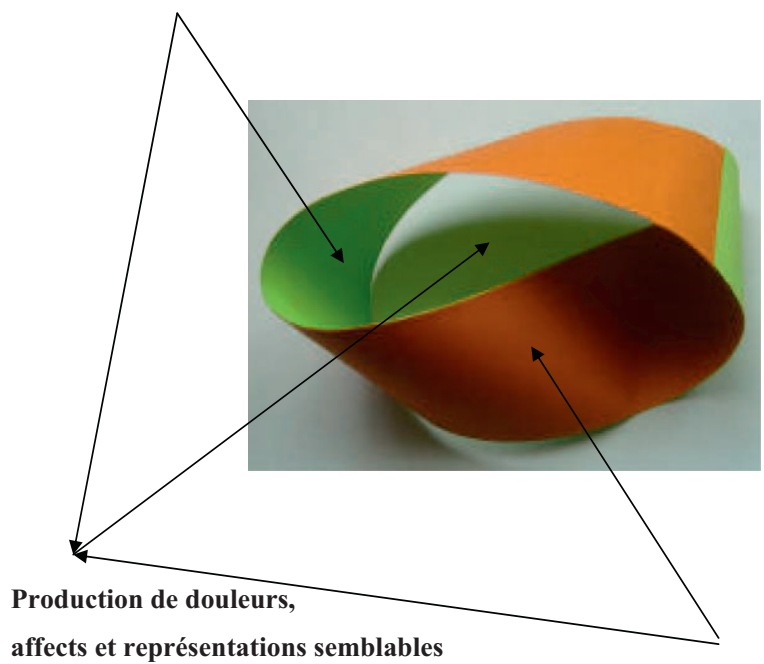

Symptômes psychiques de conversion

(douleurs lombaires, douleurs buccales)

Fig. 1.

Cette continuité et cette discontinuité peuvent être imagées par la figure topologique de la bande de Möbius ${ }^{8}$, que Lacan utilise très fréquemment au cours de ses séminaires, en particulier pour représenter les rapports du sujet et du signifiant ${ }^{9}$, la double inscription freudienne ([12], 13 janvier 1971), les relations entre les registres du conscient et de l'inconscient ${ }^{10}$, la particularité de la sexualité humaine ${ }^{11}$. La bande de Möbius ${ }^{12}$ est en effet une surface que l'on peut dire ambiguë, n'ayant qu'un seul bord et qu'une seule face, l'opposition apparente entre son endroit et son envers masquant d'autant plus la réelle continuité de sa surface ${ }^{13}$. De ce fait, par exemple, les relations complexes entre les registres du conscient et de l'inconscient en sont éclairées : il ne s'agit plus simplement de deux zones du psychisme qui seraient géographiquement séparées, mais de deux états particuliers dont les propriétés tiennent aussi à la position subjective. Dans le registre de l'hystérie, on en prendra pour illustration ce que dit Miss Lucy R. à Freud lorsque ce dernier lui fait remarquer qu'elle ne lui avait pas tout de suite révélé qu'elle était amoureuse

\footnotetext{
${ }^{8}$ Voir, pour une application développée de la figure de la bande de Möbius : Ly-Thanh-Huê, Entre contraintes et libertés, comment va le diabétique? Une approche psychanalytique de la maladie, In: ([11], p. 197-203).

9 Lacan J. Séminaire XII. Problèmes cruciaux pour la psychanalyse, (9 décembre 1964).

${ }^{10}$ Lacan J. Séminaire XXIV. L'insu que sait de l'une bévue, s'aile à mourre (13 janvier 1971).

11 Lacan J. Séminaire XXV. Le moment de conclure (14 décembre 1976).

12 La bande de Möbius, obtenue par torsion d'un demi-tour d'une bande dans l'espace, et recollement de ses extrémités, est une figure topologique à un seul bord et une seule face. Ce bord équivaut donc à un cercle. La bande de Möbius est non orientable. Couper longitudinalement une bande de Möbius ne produit pas deux bandes séparées mais un seul anneau tordu deux fois sur lui-même, qui a perdu les propriétés décrites ci-dessus. Voir à ce sujet : Granon-Lafont J., La topologie ordinaire de Jacques Lacan [13].

13 «La conjonction d'un endroit et d'un envers», dit Lacan, Lacan J. Séminaire XXV. Le moment de conclure (14 décembre 1976).
} 


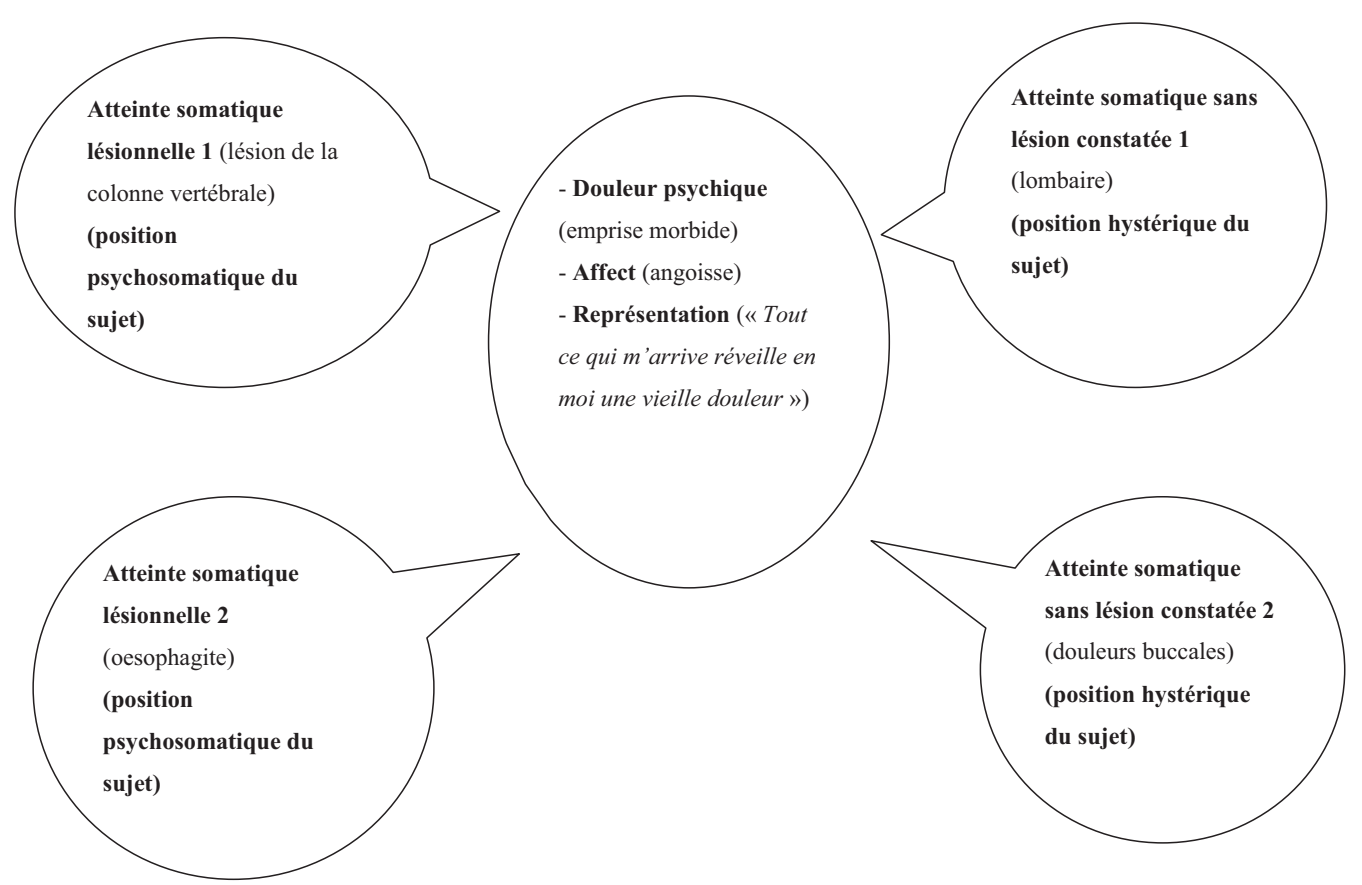

Fig. 2.

de son patron: «Mais puisque vous savez que vous aimez le directeur [son patron], pourquoi ne me l'avez-vous pas dit?»- «Je l'ignorais [répond-elle], ou plutôt je ne voulais pas le savoir, je voulais le chasser de mon esprit, et ne plus jamais y penser» ([14], p. 91).

Dans le cas qui nous occupe, celui de Loryanne, la figure de la bande de Möbius illustre bien l'entrelacement et la complexité des atteintes psychiques faisant symptôme de conversion hystérique, d'une part, et, d'autre part, les lésions corporelles ne s'inscrivant pas dans une perspective de sens et se retrouvant donc par là désubjectivées, autrement dit relevant de ce que nous appelons ici psychosomatique. Mais la figure permet aussi d'illustrer leur discontinuité clinique apparente et leur continuité subjective, leur succession temporelle et leur synchronie psychique, puisque malgré leur statut opposé, ces atteintes en arrivent à produire de mêmes douleurs, de mêmes affects et de mêmes représentations psychiques (Fig. 2 ci-dessus).

Loryanne présente donc deux types de troubles physiques, les premiers relèvent d'un processus désubjectivé, psychosomatique, les seconds sont l'expression somatique d'une difficulté psychique qu'elle parvient à élaborer. Ce qui dans son cas est remarquable réside en ce que l'élaboration des symptômes hystériques entraîne dans son sillage celle des troubles psychosomatiques. Il est donc possible d'espérer pour elle un changement de positionnement subjectif à la faveur de son travail psychothérapique.

\section{Faire surgir un symptôme}

Dans les cas que nous avons décrits, et dans de nombreux autres, les manifestations pathologiques sont donc multiples, bien que la lombalgie semble à chaque fois occuper une place centrale dans l'histoire du sujet et le discours qui tisse cette histoire. Qu'en est-il alors de la place 
du (ou des) symptômes? La diversité de la plainte ne doit pas masquer l'importance que revêt pour le sujet la centration autour d'une douleur qui devient pour lui, peut-être, plus signifiante de sa difficulté d'être, plus représentative de sa position subjective. Dans les observations que nous avons faites, c'est la lombalgie qui occupe cette fonction, et comme certains patients nous l'ont montré, nous pouvons penser alors que la douleur lombaire peut acquérir, à un moment précis, une valeur de symptôme, au sens psychanalytique du terme, alors qu' auparavant elle n'était vécue que comme un accident, autrement dit un événement aléatoire survenu dans l'existence du sujet, malgré lui, sans qu'il y soit impliqué.

Dans les entretiens, certains patients subjectivent en effet leur pathologie pour en faire l'expression d'une douleur ou d'une difficulté existentielle, et ce faisant, ils réorganisent les éléments de leur histoire pour en faire émerger les repères principaux. Contrairement à d'autres qui maintiennent leur difficulté physique dans une non-subjectivation, une extériorité psychique, qui en fait un événement morbide contingent contre lequel ils se sentent apparemment impuissants. Nous remarquerons ici que la nature même de la douleur favorise en quelque sorte ce double statut - douleur «muette», douleur « qui parle »-dans la mesure où, comme le remarque Annie Aubert, «la douleur oppose à tout effort de conceptualisation » ([15], p. 5) les plus grandes difficultés. Il s'agit alors de poser le caractère ardu, l'effort nécessaire, mais aussi certainement l'utilité de l'acte qui consiste à donner du sens à la douleur, car cet acte tend à soulager en faisant passer la douleur de son terrain réel de surgissement à celui de la parole et de l'histoire, beaucoup plus abstrait, symbolique et maîtrisable. Car le sujet tend à juguler l'envahissement douloureux qui le menace par les liens que la parole et le sens qu'elle véhicule lui offrent presque spontanément. «Actuelle et excessive, la douleur fait effraction à l'intérieur d'un appareil psychique dont elle bouleverse l'organisation» ([15], p. 212), écrit encore Annie Aubert. La douleur est un trauma, en grande partie irreprésentable et donc source d'angoisse, une «jouissance » contre laquelle on lutte par différents moyens : par sa transformation en plaisir masochiste, par l'usage d'éventuels bénéfices secondaires, mais aussi et surtout en y apposant les signifiants nécessaires à son apaisement. Car les signifiants permettent au moins d'en retirer l'excès d'angoisse : comprendre les «raisons » de la douleur la rend plus familière et permet parfois d'en imaginer plus aisément la disparition puisque, l'inscrivant dans une histoire, cela la temporalise, et ce faisant la tempère aussi.

La prise ou non en considération du symptôme par le sujet lui-même oriente l'évolution de sa pathologie, soit, dans le cas où il refuse de s'y reconnaître comme acteur, en l'enkystant dans un statut de phénomène psychosomatique quasi inaccessible à l'analyse mais nécessaire à son fonctionnement psychique, soit, lorsqu'il « admet y être », en lui permettant de faire surgir d'autres représentations qui déplacent le problème et lui donnent alors accès à une voie d'élaboration où la douleur «primaire » de la lombalgie est relativisée et devient moins «nécessaire ».

La psychosomatique n'est donc, dans notre perspective, ni un type particulier de maladies, ni une structure psychique différenciée, mais un positionnement du sujet susceptible d'être modifié.

\section{Conclusion}

Dans le cadre d'une consultation en médecine générale, il serait illusoire de considérer que l'on puisse amener tous les patients à faire de leur lombalgie un symptôme, au sens psychanalytique du terme. En effet, le cadre thérapeutique est un élément constitutif du type de transfert qui s'établit entre un patient et un praticien, si bien que le transfert en médecine générale ne vise pas les mêmes effets que le transfert en psychothérapie ou en psychanalyse. La relation médecin-malade en médecine générale favorise «l'établissement d'un transfert original » dit Louis Velluet [16]. Un patient qui va consulter son médecin n'en attend pas la même chose que le patient qui va consulter 
un psychologue, un psychanalyste ou un psychiatre. La formation du praticien est également en cause $^{14}$. Cependant, l'ouverture des uns et des autres à la possibilité d'une évolution du patient fondée sur un changement de sa position subjective peut être un avantage tout à fait important. Les médecins généralistes pourraient ajouter à l'exploration sémiologique habituelle ce volet nouveau qui fait apparaître les capacités du patient à subjectiver, ce qui permet d'accéder ainsi au champ psychothérapique. Ce qui est en jeu dans le processus de la chronicisation n'est pas seulement la disparition pure et simple de la douleur, mais la modification préalable de sa fonction pour le sujet, c'est-à-dire le passage de la fonction première de la douleur comme signal de danger à celle qui favorise l'élaboration d'un sens subjectif. Et pour cela, faut-il encore que ce sujet, comme les praticiens concernés, lui reconnaissent cette seconde fonction, pouvant, on le sait, être saisie à différents niveaux, souvent intriqués, un niveau clinique, un niveau «travail », un niveau psychologique et social ${ }^{15}$. Les obstacles cliniques, les bénéfices secondaires d'une invalidité, ne se traitent pas de la même manière que les obstacles psychiques ou sociaux. Néanmoins nous soutenons que ces derniers obstacles ne font jamais défaut et qu'envisager seulement de pouvoir agir sur eux représente déjà une amélioration potentielle de la prise en charge et un espoir de mieux-être pour le patient.

\section{Conflit d'intérêt}

Les auteurs déclarent n'avoir aucun conflit d'intérêt.

\section{Références}

[1] Poiraudeau S, Lefèvre-Colau M-M, Fayad F, Rannou F, Revel M. Lombalgies Encycl Méd Chir (Elsevier Paris). Rhumatol Orthoped 2004;1:295-319.

[2] Agence Nationale d'Accréditation et d'Évaluation en Santé. Service des recommandations et références professionnelles. Diagnostic, prise en charge et suivi des malades atteints de lombalgie chronique; décembre 2000 : Available from: http://www.has-sante.fr/portail/jcms/c_271859/diagnostic-prise-en-charge-et-suivi-des-maladesatteints-de-lombalgie-chronique.

[3] Benoist V, Martin-Mattera P, Ly-Thanh-Huê H, Huez JF, Garnier F. La chronicité chez les patients lombalgiques. Synoviale 2009;172:19-21.

[4] Freud S. Euvres complètes, T. XIV. Paris: PUF; 2000.

[5] Keller PH. Médecine et psychosomatique. Paris: Flammarion; 2000.

[6] Sifneos P. The Prevalence of "alexithymia" characteristics in psychosomatic patients. Topics of psychosomatic research. Bâle: Karger; 1972.

[7] Marty P, De M’Uzan M. La pensée opératoire. Rev Fr Psychanal 1963;27(SP):345-56.

[8] Marty P. Les mouvements individuels de vie et de mort. Paris: Payot; 1976.

[9] Sami-Ali M. Corps réel, corps imaginaire. Paris: Dunod; 1977.

[10] Guir J. Psychosomatique et cancer. Paris: Point Hors ligne; 1984.

[11] Ly-Thanh-Huê H. Entre contraintes et libertés, comment va le diabétique ? Une approche psychanalytique de la maladie. Diabete Obesite 2007;10:197-203.

[12] Lacan J. Séminaire XVIII. D’un discours qui ne serait pas du semblant. Paris: Seuil; 2006.

[13] Granon-Lafont J. La topologie ordinaire de Jacques Lacan. Paris: Point Hors Ligne; 1985.

[14] Freud S. Études sur l'hystérie. Paris: PUF; 1956.

\footnotetext{
14 Voir par exemple Balint M. et Balint E. ([17], p. 169). «Les hôpitaux universitaires [devraient] proposer aux étudiants une formation sérieuse à la psychothérapie ». Balint traitait déjà à la fin des années 1950 d'une question que la législation de 2009 en France (voir les difficultés de l'article 52 de la loi du 9 août 2004) peine encore à résoudre : celle de la formation à la psychothérapie.

15 Voir Ly-Thanh-Huê [18].
} 
[15] Aubert A. La douleur. Paris: PUF; 1996.

[16] Velluet L. La psychothérapie spécifique du médecin généraliste. Exercer 2001;(60):4-10.

[17] Balint M, Balint E. Techniques psychothérapeutiques en médecine. Paris: Payot; 1970.

[18] Ly-Thanh-Huê. Avoir le diabète ou être diabétique ? Rôles et limites des groupes de diabétiques. Diabete Obesite 2007; 12:245-50. 